\title{
Anisotropic properties of elastomeric nanocomposites based on natural rubber and $\mathrm{sp}^{2}$ carbon allotropes
}

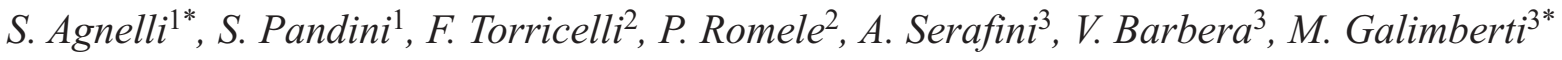 \\ ${ }^{1}$ Department of Mechanical and Industrial Engineering, University of Brescia, Via Branze 38, 25123 Brescia, Italy \\ ${ }^{2}$ Department of Information Engineering, University of Brescia, Via Branze 38, 25123 Brescia, Italy \\ ${ }^{3}$ Department of Chemistry, Materials and Chemical Engineering G. Natta, Politecnico di Milano, via Mancinelli 7, \\ 20131 Milano, Italy
}

Received 23 December 2017; accepted in revised form 16 March 2018

\begin{abstract}
This work provides a comprehensive investigation of the anisotropic mechanical and electrical properties of elastomeric nanocomposites based on natural rubber and $\mathrm{sp}^{2}$ carbon allotropes. They can be either nanometric and with high shape anisotropy like Carbon Nanotubes (CNT) and lamellar nanographite, or nanostructured and nearly isometric like carbon black. Studies were performed on calendered and compression molded plates. A complete mechanical characterization along all main directions could be performed by a non-standard testing approach. Composites with nanometric, high aspect ratio fillers gave rise to remarkable mechanical anisotropy, revealing an orthotropic and transversally isotropic response: modulus values were very similar in the sheet plane and much larger (almost twice as much) in the orthogonal direction. The electrical anisotropy achieved its maximum at lower CNT content. Composites with carbon black did not reveal mechanical anisotropy, while, quite strikingly, a very large electrical anisotropy was observed for carbon black content close to the percolation threshold. These results provide insights into the anisotropic behavior of nanofilled elastomers, and could pave the way to their exploitation in advanced engineering design and biomimicking biomedical applications.
\end{abstract}

Keywords: nanocomposites, mechanical anisotropy, electrical anisotropy

\section{Introduction}

Important load bearing applications require elastomeric materials, in many different fields. Rubbers are used, for example, for tire treads, vibration dumpers, seals. Although much softer than rubber, tough and highly deformable hydrogels are being developed and have reached the status of structural materials in applications such as cartilage replacement, scaffolds for tissue engineering or supports for mechanobiology studies. For both classes of materials, the addition of reinforcing fillers is an effective route to promote the mechanical reinforcement $[1$, 2]. $\mathrm{sp}^{2}$ carbon allotropes are traditional reinforcing fillers. Carbon black is the most used [3, 4] and nanofillers have been increasing their importance over the last years, both in the academic and industrial fields [5]. Nanofillers are particles with at least one dimension below $100 \mathrm{~nm}$ [6]. Some examples are Carbon Nanotubes (CNT), both single-walled and multiwalled [6-8], graphene and graphene related materials such as graphene nanoplatelets made by few stacked layers of graphene (nanoG) [9-12]. Carbon black is formed by primary nanometric particles, fused together to form larger aggregates, which can reach a size of the order of micrometers. Instead, the nanofiller particles can be individually dispersed in the elastomeric matrix. This results in a very high surface to volume ratio and, as a consequence, in a higher reinforcing efficiency with respect to carbon black content. 
A common rationalization of mechanical reinforcement by different families of $\mathrm{sp}^{2}$ carbon allotropes has been recently proposed [13-15]. A quantitative correlation has been shown between the initial moduli of rubber based composites filled with carbon black or CNT, and the interfacial area, that means the area made available by the filler in the elastomeric composite.

The high surface area of nanometric carbon allotropes originates not only from their small dimensions, but also from their high aspect ratio (or shape anisotropy), i.e. the ratio of the maximum over the minimum dimension of the particle [16, 17]. Owing to this feature, the particles may present a preferential orientation in the elastomeric matrix and, as a consequence, the composite may show anisotropic properties, that means directionally dependent properties. Anisotropic properties in elastomeric matrices may be caused by several reasons: i) orientation of the polymer chains; ii) non homogeneous filler distribution, even with ideally perfectly spherical particles; iii) stress softening caused by a repeated stretching (Mullins effect) in one direction [18-21]; iv) preferential orientation of anisotropic filler particles [22, 23].

Preferential orientation and alignment of nanoparticles is sought to fully exploit their influence along specific directions, as for example in electronical applications (e.g. field emission displays and sensors, data storage, and light-emitters) with CNT filled elastomers [24], or in biomimicking, tissue engineering, and bioseparation applications, where hydrogels are filled with CNT [25] or nanowhiskers [26] to induce direction-dependent structure with the aim to mimic anisotropic hierarchical morphologies of some natural tissues.

Indeed, the control of anisotropic properties of elastomeric materials is of great importance. Modeling of composites with electrically conductive nanorods has been recently presented [27]. However, only few experimental works $[22,28]$ provide a measure of properties along transversal directions and a quantification of the anisotropy level. A remarkable anisotropic behavior of the dynamic moduli has been documented for CNT filled Natural Rubber (NR) [22]. The structuring of CNT induced an orthotropic and transversally isotropic response. As a result, the dynamic moduli were very similar to each other when measured inside the sheet plane and nearly double when measured in the orthogonal direction (i.e. perpendicularly to the sheet plane). Moreover, Transmission Electron Microscopy (TEM) analyses showed a preferential orientation of CNT inside the natural rubber based composites.

In spite of the scientific and applicative impact of this finding, there is a lack of systematic studies on the effect of filler aspect ratio on the level of anisotropy of rubber composite properties.

In the present work, a comprehensive investigation and analysis of the anisotropic behavior of rubber nanocomposites filled with $\mathrm{sp}^{2}$ carbon allotropes having different shape anisotropy has been performed. Although the results are specific for the considered materials, the experimental testing approach has a more general validity, and could be applied both to rubber and hydrogels, produced with different processes. Mechanical and electrical properties have been studied. Three classes of carbon-based fillers with very different shape anisotropy were accounted for: multiwalled carbon nanotubes, nanographite, and two types of carbon black with different level of surface area. The nanographite is characterized by a high surface area and relatively high shape anisotropy [29]. One of the two carbon black is N326 (CB-MS), having an average (medium) level surface area and structure, whereas the other one is characterized by a very high surface area (CB-HS). Carbon black was investigated since it shows low or even zero effects of anisotropy, providing a sort of lower limit of anisotropy level for comparison purposes.

The mechanical and electrical properties of elastomeric composites were investigated along three main spatial directions of a rubber plate that is a simple, yet relevant rubber product. The plate allowed to take advantage of the intrinsic filler orientation induced by the production process, calendering and compression molding. The anisotropic mechanical behavior was measured in terms of dynamic-mechanical moduli along various directions, evaluating the moduli dependence on the strain amplitude. A complete mechanical characterization could be performed by the suitable choice of specimen size and test machine, an ad-hoc approach developed by the authors. To further evidence filler-related anisotropic effects, the electrical resistivity was measured along the same directions. The correlation of such properties with structural morphology of the nanocomposites was investigated by transmission electron microscopy. 


\section{Experimental}

\subsection{Materials}

Poly(1,4-cis-isoprene) from Hevea Brasiliensis (NR) was SMR GP, with 65 Mooney units as Mooney viscosity $\left(\mathrm{ML}(1+4) 100^{\circ} \mathrm{C}\right)$, from BR-THAI, Eastern GR Thailandia - Chonburi, Lee Rubber.

Organic peroxide was 2,5-Dimethyl-2,5-di(tert-butylperoxy)hexane supported on silica/ $\mathrm{CaCO}_{3}$ (45 mass\% of peroxide), from Arkema Inc (King of Prussia, PA, USA).

Four kinds of fillers were used: CNT, nanoG, and two kinds of CB-MS.

Multiwall carbon nanotubes are Baytubes C150 P, from Bayer Material Science (Leverkusen, Germany). They are characterized by chemical purity $\geq 95 \mathrm{wt} \%$, length of $1-10 \mu \mathrm{m}$, number of walls of $3-15$, outer and inner diameters of 10-16 and $4 \mathrm{~nm}$, respectively, according to the technical data sheet. Their surface area, measured by the BET method, is $200 \mathrm{~m}^{2} / \mathrm{g}$. The following safety procedure was followed to avoid the direct contact of the operator with CNT. They were kept and weighed in a glovebox. The ingredients were fed in the mixing chamber through a steel funnel on the top. The mixing chamber was not open to the air during mixing.

Carbon Black N326 (CB-MS) is from Cabot Corporation (Billerica, MA, USA), and is characterized by $30 \mathrm{~m}$ as mean diameter of spherical primary particles, surface area, measured by the BET method, of $77 \mathrm{~m}^{2} / \mathrm{g}$ and oil adsorption number (with Dibutyl Phthalate) of $85 \mathrm{~mL} / 100 \mathrm{~g}$.

Carbon Black PRINTEX XE2 (CB-HS) is from Degussa (The Cary Company, Addison, IL, USA) and it is characterized by a surface area, measured by the BET method, of $1114 \mathrm{~m}^{2} / \mathrm{g}$.

NanoGraphite (nanoG) is Synthetic Graphite $8427^{\circledR}$ from Asbury Graphite Mills Inc (Asbury, NJ, USA). Its carbon content is $\geq 99 \mathrm{wt} \%$ and the surface area is $330 \mathrm{~m}^{2} / \mathrm{g}$ (data from technical bulletin). Chemical composition determined from elemental analysis is, as $\mathrm{wt} \%$ : carbon 99.5 , hydrogen 0.4 , nitrogen 0.1 , oxygen 0.0 . NanoG is characterized by a high shape anisotropy compared to several graphite grades. A value of 3.1 was measured for the shape anisotropy defined as the ratio between crystallites dimensions in directions orthogonal and parallel to structural layers [29].

\subsection{Composites preparation}

All the composites were produced by adding different amounts of filler, namely 4,15 or $35 \mathrm{phr}$ (parts per hundred parts of rubber), to $100 \mathrm{phr}$ of NR and $3.5 \mathrm{phr}$ of peroxide. Peroxide was used as the crosslinking agent in order to avoid the addition of several ingredients that would be required by a sulfur-based cross-linking system. The filler amounts both in phr and in volume fraction are reported in Table 1. Additionally, also a sample of neat crosslinked NR rubber and a sample filled with $50 \mathrm{phr}$ of CB-MS (CBMS-50) were prepared.

\section{Preparation procedure}

Composites were prepared by mechanical mixing in a Brabender internal mixer (Brabender PL-2000 Plasti-Corder Torque Rheometer, Brabender GmbH \& Co. KG, Duisburg, Germany), with $50 \mathrm{~mL}$ mixing chamber. The fill factor of the mixing chamber was about $80 \%$. The ingredients were added in the following sequence: NR was masticated at $80^{\circ} \mathrm{C}$ for $1 \mathrm{~min}$ with rotors at $60 \mathrm{rpm}$. The filler was then added and mixed for further $4 \mathrm{~min}$; then peroxide was added and the final composite was discharged after $2 \mathrm{~min}$. The mixing time, shorter than the half-life of peroxide $\left(10 \mathrm{~h}\right.$ at $\left.115^{\circ} \mathrm{C}\right)$, was set to avoid premature crosslinking, which was not observed. Composites were finally further homogeneized by passing them five times through a two roll mill operating at $50^{\circ} \mathrm{C}$, with the front roll rotating at $30 \mathrm{rpm}$ and the back roll rotating at $38 \mathrm{rpm}$ and $2 \mathrm{~mm}$ as the nip between the rolls. The sheet of the compounds was rotated at every step. Finally the sheets were compression molded for $10 \mathrm{~min}$ at $170^{\circ} \mathrm{C}$ with $3.5 \mathrm{MPa}$ pressure into square plates with $3 \mathrm{~mm}$ thickness and $100 \mathrm{~mm}$ edge.

Table 1. Recipes of composites based on NR and carbon nanofillers ${ }^{\mathrm{a}}$.

\begin{tabular}{|l|c|c|c|}
\hline \multicolumn{1}{|c|}{ Filler type } & \multicolumn{3}{|c|}{ Composite $^{\text {b }}$} \\
\hline \multirow{2}{*}{ CNT } & CNT-4 & CNT-15 & CNT-35 \\
& $4(0.02)$ & $15(0.07)$ & $35(0.15)$ \\
\hline \multirow{2}{*}{ nanoG } & nanoG-4 & nanoG-15 & nanoG-35 \\
& $4(0.02)$ & $15(0.07)$ & $35(0.15)$ \\
\hline \multirow{2}{*}{ CB-MS } & CB-MS-4 & CB-MS-15 & CB-MS-35 \\
& $4(0.02)$ & $15(0.07)$ & $35(0.15)$ \\
\hline \multirow{2}{*}{ CB-HS } & & & CB-HS-35 \\
& & & $35(0.15)$ \\
\hline
\end{tabular}

${ }^{\mathrm{a}}$ Other ingredients: NR $100 \mathrm{phr}$, peroxide $1.58 \mathrm{phr}$ (peroxide/sili$\mathrm{ca} / \mathrm{CaCO}_{3} 3.5 \mathrm{phr}$ ),

${ }^{\mathrm{b}}$ Are reported: label of the composite (e.g.: CNT-4), the amount in phr and, in brackets, the volume fraction of carbon filler. 


\subsection{Dynamic-mechanical analyses}

\subsubsection{Samples preparation}

Parallelepiped specimens were cut from the square sheets of cured rubber. The nominal dimensions of the specimens were: height $=6 \mathrm{~mm}$, width $=$ thickness $=3 \mathrm{~mm}$ (see Figure 1a). The actual dimensions of each specimen in its unstrained state were measured before testing by a travelling optical microscope.

\subsubsection{Measurements}

Dynamic mechanical tests were carried out by a dynamic-mechanical analyzer Q800 (TA Instruments, New Castle, DE, USA). All tests were performed at room temperature and $1 \mathrm{~Hz}$, in strain sweep mode. The strain amplitude ranged from a minimum of $0.02 \%$ up to the maximum strain level that may be applied according to the machine bearing capabilities (maximum load: $18 \mathrm{~N}$ ).
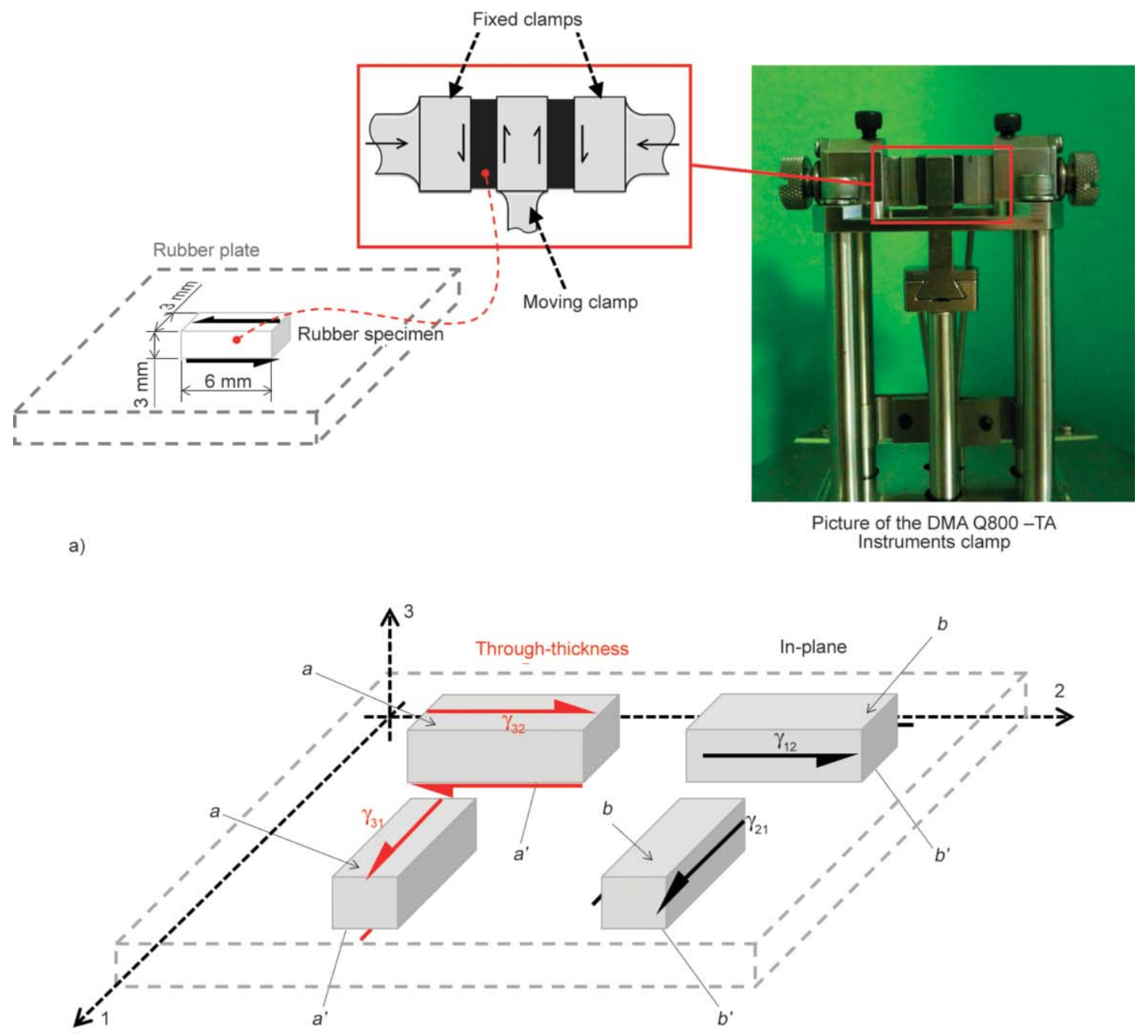

b)

c)

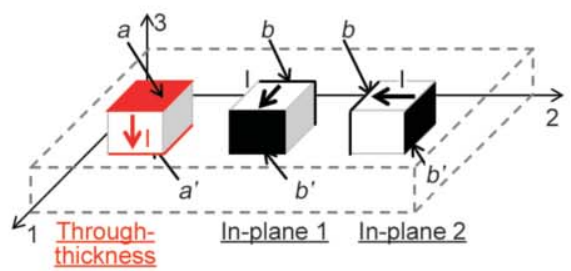

Figure 1. Schematic representation of test configurations. a) Parallelepiped samples were taken from a crosslinked nanocomposite sheet and tested in the shear sandwich configuration by dynamic-mechanical tests. b) Representation of direction of shear strains applied to specimens cut from the rubber sheets (represented by the dashed lines) in 'through-thickness' and 'in-plane' configurations; the coordinates of the reference system are also displayed. c) Representation of the cubic specimens, in TT and IP configuration, and the corresponding current flow (solid arrows) for the electrical measurements. 
The shear sandwich configuration used for testing is shown in Figure 1a.

In the shear sandwich configuration, two nominally identical parallelepiped rubber specimens were inserted between two outer plates. A central, moving clamp separated the two specimens. The specimens were slightly compressed by the two outer clamps (a compressive strain of about $15 \%$ was employed) in order that friction forces guarantee no slippage of the specimens on the clamps.

Before each test, the specimens were mechanically conditioned: a strain sweep $(0.02 \%$-maximum shear strain amplitude, $1 \mathrm{~Hz}$ ) was applied and an equilibration phase followed $(0.02 \%$ shear strain amplitude, $1 \mathrm{~Hz}$, for $15 \mathrm{~min}$ ). Finally, the dynamic moduli were recorded as a function of strain amplitude during the test. At least three repetitions were performed for each test.

\subsubsection{Test configurations}

Figure $1 \mathrm{~b}$ schematically depicts the orientation of the specimens and of the strain directions with respect to the rubber sheet, represented by the dashed lines. The specimens, represented as parallelepipeds drawn with the solid line, can be oriented along two main directions perpendicular with respect to each other. A Cartesian coordinate system helps to define the loading direction of the specimens. The two main dimensions of the rubber sheet were arbitrarily labelled with axes 1 and 2. Plane 1-2 thus defines the main plane of the rubber sheet. Perpendicularly to the sheet main plane is axis 3 , which crosses the $3 \mathrm{~mm}$ thickness. With reference to the sheet processing, axis 3 refers also to the direction of application of the pressure in the roll milling and in the molding process. Instead no reference to specific processing direction can be done with axis 1 and 2, since during milling the rubber sheet was rotated at every step and the mold configuration is symmetrical. Four test configurations (see Figure 1b) were performed to study the material response in different directions. Such configurations can be divided in two groups: 'throughthickness' (TT) and 'in-plane' (IP), labelled with reference to basic definitions of composites laminate [30], widely used also for fiber-reinforced polymer composites. For both TT and IP configurations, two specimens, perpendicularly oriented to each other, were tested. In 'through-thickness' (TT) tests the shear stress was applied on the $a$ and $a^{\prime}$ faces of the sample, along axis 1 or 2 , and the shear deformation developed through the sample thickness. TT configuration allowed to measure $G_{31}$ and $G_{32}$ moduli, by the application of $\gamma_{31}$ and $\gamma_{32}$ shear strains, respectively. In 'in-plane' (IP) tests the shear stress was applied on $b$ and $b^{\prime}$ faces of the sample, along axis 1 or 2 , and the shear deformation developed in the 1-2 plane. IP configuration allowed to measure $G_{12}$ and $G_{21}$ moduli, by the application of $\gamma_{12}$ and $\gamma_{21}$ shear strains, respectively.

\subsection{Bright field transmission electron microscopy (BF-TEM) and selected area electron diffraction (SAED) analyses}

The structuring of the filler in nanoG-15 and nanoG35 samples was carried out by TEM analyses. BFTEM analysis was coupled with Selected Area Electron Diffraction (SAED) analysis. BF-TEM micrographs and SAED patterns were acquired using a Philips CM200 electron microscope operating at $200 \mathrm{kV}$ equipped with a Field Emission Gun filament (FEI Company, Eindhoven, The Netherlands). A Gatan US 1000 CCD camera (Pleasanton, CA, USA) was used and $2048 \times 2048$ pixels images with 256 grey levels were recorded. Thin film cross-sections (approximately $70-100 \mathrm{~nm}$ thick) were prepared at $-130^{\circ} \mathrm{C}$ using a Leica EM FCS cryo-ultramicrotome (Wetzlar, Germany) equipped with a diamond knife and mounted onto 300 mesh copper grids. No heavy metal staining methods were used. Analyzed sections were thus oriented parallel to axis 3 (shown also in Figures $1 \mathrm{~b}$ and 1c) and allowed to observe the nanocomposite through the sheet thickness.

\subsection{Electrical resistivity measurements}

The electrical measurements were performed in a Janis ST-500 micro-manipulated probe station (Janis, MA, USA) under ambient conditions and shielded against electromagnetic interferences. The specimens were contacted with the probe-station tips and connected to the instruments with triaxial cables. In order to ensure a good electrical contact between the specimen and the tips, two of the six surfaces of the specimen were covered with high-conductivity silver paste. Current-voltage $(I-V)$ electrical measurements were performed using a KEITHLEY 2636A SourceMeter Unit (SMU) (Keithely, a Tektronics company, Beaverton, OR, USA). The SMU was programmed and controlled with a computer by using MATLAB. The measurement system (viz. micro-manipulated probe station with the SMU) can measure currents 
as low as $10^{-13} \mathrm{~A}$ with a voltage resolution of $5 \cdot 10^{-6} \mathrm{~V}$. The $I-V$ characteristics were measured on cubic specimens with a volume of about $3 \mathrm{~mm}^{3}$. The voltage was swept from -5 to $+5 \mathrm{~V}$ and the current was recorded. We calculated the resistance as the best fit linear interpolation of the $I-V$ characteristic (i.e. interpolation function $\hat{I}=V / R$ where $R$ is the resistance) and then the bulk resistivity results: $\rho=R \cdot S / h$ where $S$ and $h$ are the surface area and thickness of the specimen, respectively. The reliability of the bulk resistivity measurement was checked. In fact, preliminary tests performed on specimens with different dimensions showed that the measured resistance scales linearly with the specimen thickness, and this indicates that it is not due to the surface conduction. Two main test configurations were used. In the 'through-thickness' configuration, the current flow was measured along axis 3 , i.e. across the thickness, as shown in Figure 1c. In the 'in-plane' configuration the current flow was measured in the plane of the plate, along axis 1 or 2, as shown in in Figure 1c. The actual dimensions of the specimens, that means the thickness and the facet area, were measured by using a calibrated digital microscope (Dino-Eye AM7023CT-LH1 DinoEye, Naarden, The Netherlands).

\section{Results and discussion}

This work studies the level of mechanical and electrical anisotropy promoted by carbon based fillers with different shape anisotropy, in crosslinked NR based composites. As reported in the introduction, the carbon allotropes were a medium and a high surface area carbon black with nearly isotropic particles and anisometric nanofillers, such as a high surface area nanosized graphite and carbon nanotubes. Such fillers differ also for their aspect ratio. Only average values can be estimated. From TEM images of CNT filled NR systems (reported in [22]), the aspect ratio of CNT can be estimated to be larger than 10. In the case of nanoG, as reported in the experimental part, XRD analysis allowed to determine the anisotropic index $[11,29]$, which was found to be 3.1. However, this value is based on the size of crystalline domains. As the lateral size of nanoG lamella is larger than the size of the crystalline domain (it can be in the range from $300 \mathrm{~nm}$ to more than $500 \mathrm{~nm}$ ), the aspect ratio can also be larger than 10. The aspect ratio of CB-MS and CB-HS can be hypothesized to be not much larger than 1 . Therefore, the filler shape anisotropy follows the ranking: $\mathrm{CNT} \geq$ nanoG $>\mathrm{CB}-\mathrm{MS}=\mathrm{CB}-\mathrm{HS}$.

\subsection{Dynamic-mechanical tests}

The shear sandwich configuration allowed to test the mechanical material properties both in the plane of the molded sheets (IP configuration) and orthogonally (TT configuration). Figure 2 schematically shows examples of such configurations, with the illustration of the possible structuring of the fillers inside

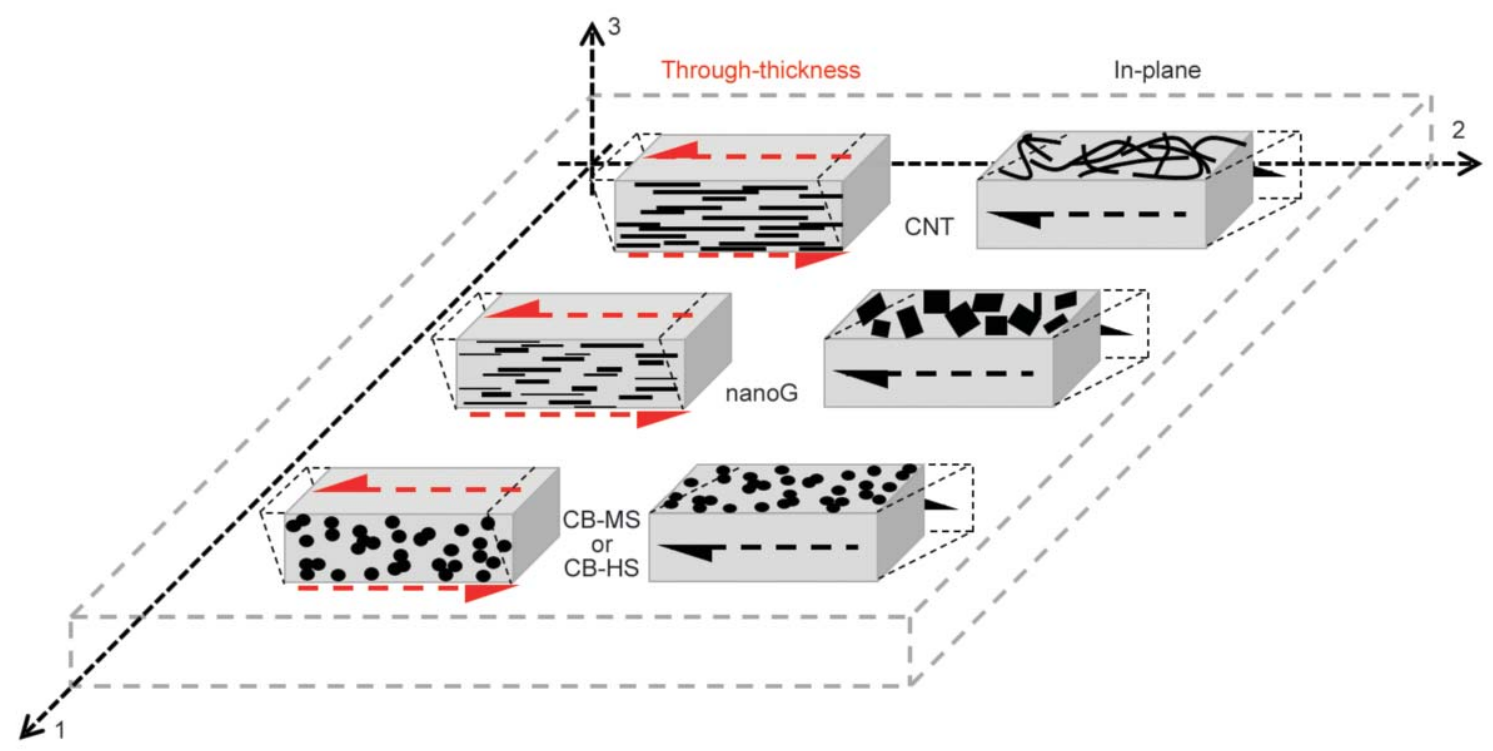

Figure 2. Schematic representation of some examples of dynamic-mechanical test configurations. Shear strains, indicated by dashed arrows, were applied in 'through-thickness' $\left(\gamma_{31}, \gamma_{32}\right)$ (specimens on the left), and in 'in-plane' tests $\left(\gamma_{12}\right.$, $\gamma_{21}$ ) (specimens on the right) on parallelepiped specimens (grey shapes). CNT, nanoG and CB-MS expected organization is schematically sketched with a lateral view in 'through-thickness' tests and with a top view in 'inplane' tests. 
the rubber plate, which will be discussed below in the text.

The mechanical dynamic moduli were measured over a range of strain amplitudes. Filled rubbers typically showed a nonlinear behavior, i.e. a reduction of the modulus with strain amplitude. Such phenomenon, known as Payne effect [31], is related to dissipation of energy in dynamic-mechanical loading of elastomeric materials. The intensity of the Payne effect reflects the filler networking phenomenon, according to models which refer to two main interpretations: agglomeration-de-agglomeration process of the filler network above the filler percolation threshold [31-34] or polymer-filler bonding and debonding [35-43]. The reduction of modulus is correlated with the increase of the maximum value of loss modulus $G^{\prime \prime}$. Such parameter is reported in the results as an indication of energy dissipation of the investigated composites.

In the following, the anisotropic effects promoted by the different carbon fillers, at $35 \mathrm{phr}$ loading, are compared. The dependence of the anisotropic effects on the filler content is then discussed.

\subsubsection{Effect of filler type (at $35 \mathrm{phr}$ loading) on anisotropic behavior of NR based composites}

Figure 3 shows the storage moduli, i.e. $G_{12}, G_{21}, G_{31}$, $G_{32}$, versus the shear strain amplitude for composites with carbon fillers at $35 \mathrm{phr}$ loading. Measurements were carried out on at least three different specimens for each testing direction. Curves of CNT-35 were obtained and reported in [22]. In the present paper, only two representative curves of CNT-35 tested in TT $\left(G_{31}\right)$ and in IP $\left(G_{21}\right)$ configuration are reported. They are compared in Figure $3 \mathrm{a}$ with the curves of CB-HS-35, as these composites achieve the largest values of moduli.

The average values of shear storage modulus $G^{\prime}$ at low $(0.025 \%)$ shear strain amplitude $\left(G^{\prime}{ }_{0.025} \%\right.$, and of $G^{\prime \prime}{ }_{\max }$, both in IP and TT configuration are reported in Table 2.

Graphs in Figure 3 and data in Table 2 reveal that CNT, CB-HS, nanoG and CB-MS promote different levels of modulus, modulus nonlinearity and mechanical anisotropy, for the same filler content. Considering the average of moduli values in IP configuration ( $G_{12}$ and $G_{21}$ moduli), CNT and CB-HS reach the highest level of moduli at low strain amplitude (up to more than $20 \mathrm{MPa}$ ), remarkably higher than what
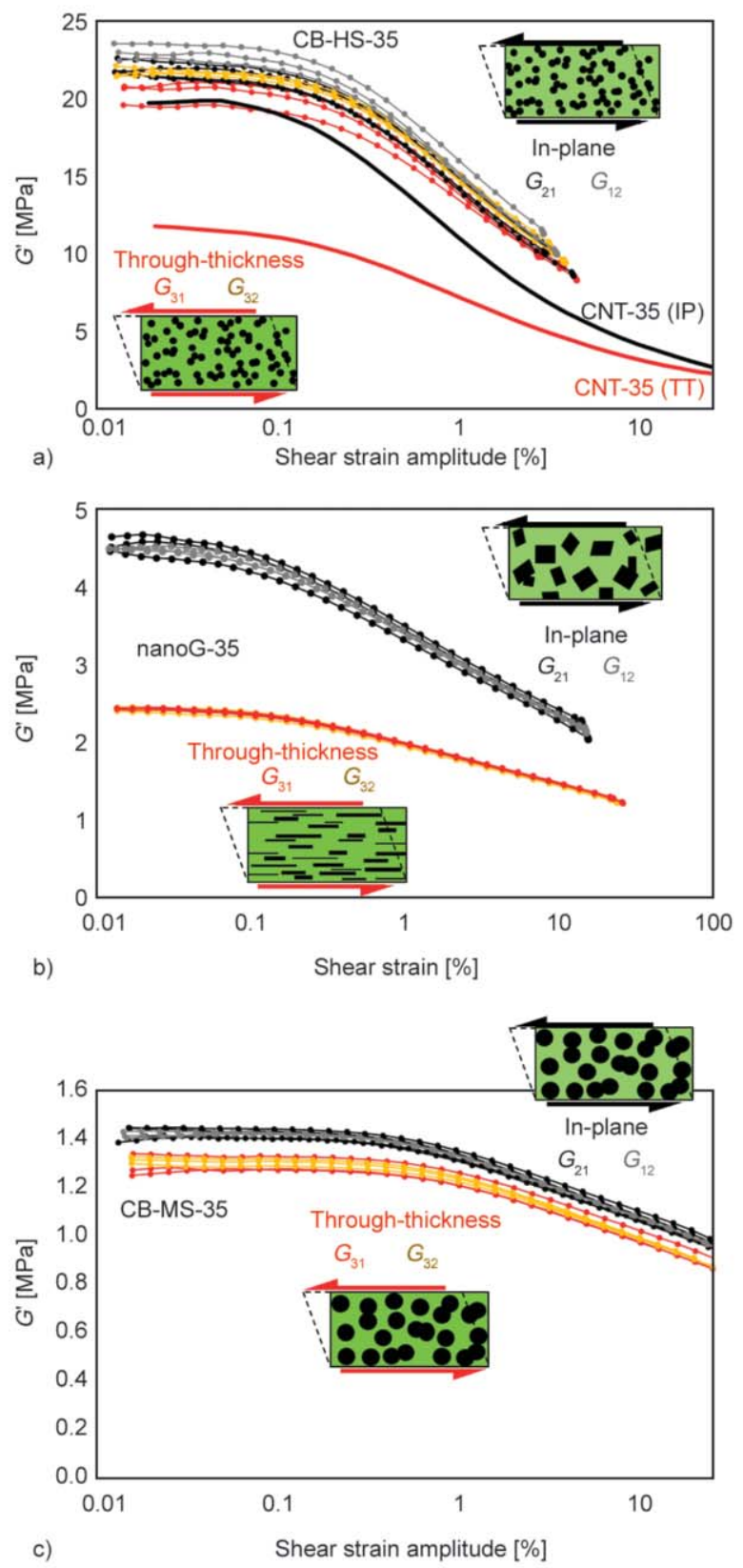

Figure 3. Storage moduli, $G_{12}, G_{21}, G_{31}, G_{32}$, vs shear strain amplitude, obtained in different test configurations for composites filled with $35 \mathrm{phr}$ of carbon fillers. Curves refer to the carbon fillers indicated as follows. a) CB-HS and CNT. Only two representative curves, TT- $G_{31}$ and IP- $G_{21}$ (taken from ref. [22]) are shown for CNT. Composite pictures refer only to $\mathrm{CB}-\mathrm{HS}$, for $\mathrm{CNT}$ see Figure 2. b) nanoG. c) CB-MS.

was obtained with nanoG (4.4 MPa) and CB-MS (1.42 MPa). This effect could be explained by the higher BET surface area of CNT and CB-HS. At the same filler content, a large surface area enhances the interfacial area with the polymer, thus increasing the reinforcing effect. The role of interfacial area as the main parameter governing the reinforcing effects 
Table 2. Average values of storage modulus $G^{\prime}$ at minimum $(0.025 \%)$ shear strain amplitude and of maximum value of loss modulus $\left(G^{\prime \prime}{ }_{\max }\right)$, both in IP and TT configuration, and of anisotropy index for the composites with 35 phr filler.

\begin{tabular}{|l|c|c|c|c|}
\hline & CNT-35 $^{*}$ & nanoG-35 & CB-MS-35 & CB-HS-35 \\
\hline$G_{\text {IP, } 0.025 \%}^{\prime}[\mathrm{MPa}]$ & $20.5 \pm 1.8$ & $4.40 \pm 0.07$ & $1.422 \pm 0.017$ & $22.2 \pm 0.8$ \\
\hline$G^{\prime}{ }_{\mathrm{TT}, 0.025 \%}[\mathrm{MPa}]$ & $10.5 \pm 0.9$ & $2.4 \pm 0.2$ & $1.299 \pm 0.027$ & $21.0 \pm 0.9$ \\
\hline Anisotropy index $\left(G_{0.025 \%}^{\prime}\right)$ & $1.95 \pm 0.06$ & $1.83 \pm 0.05$ & $1.10 \pm 0.02$ & $1.06 \pm 0.05$ \\
\hline$G_{\text {max } \text { IP }[\mathrm{MPa}]}^{\prime \prime}$ & $3.4 \pm 0.3$ & $0.495 \pm 0.006$ & $0.139 \pm 0.003$ & $2.68 \pm 0.08$ \\
\hline$G_{\text {max } \text { TT }[\mathrm{MPa}]}$ & $1.66 \pm 0.07$ & $0.27 \pm 0.01$ & $0.129 \pm 0.003$ & $2.54 \pm 0.07$ \\
\hline Anisotropy index $\left(G^{\prime \prime}{ }_{\text {max }}\right)$ & $2.07 \pm 0.04$ & $1.86 \pm 0.03$ & $1.08 \pm 0.03$ & $1.05 \pm 0.04$ \\
\hline
\end{tabular}

*data from ref [22]

promoted, at small strain by carbon fillers has already been highlighted [13, 15-17]. Although the nanoG specific surface area measured by BET $\left(330 \mathrm{~m}^{2} / \mathrm{g}\right)$ is higher than that of CNT $\left(200 \mathrm{~m}^{2} / \mathrm{g}\right)$, the reinforcing effect of nanoG is lower than that of CNT. In previous works by some of the authors $[13,15]$ it was commented that, in the case of nanoG, the surface area measured by BET method (via nitrogen absorption) is not completely available to the polymer chains: graphene layers are prevailingly stacked in crystalline domains. The ability of carbon fillers to promote high values of $\left(G^{\prime \prime}{ }_{\max }\right)$ seems to follow the same order already observed for the storage modulus: CNT > CB-HS > nanoG $>$ CB-MS. CNT and CB-HS show a $G^{\prime \prime}{ }_{\text {max }}$ an order of magnitude higher than the other fillers.

Figure 3 also shows that the filler surface area is not the only parameter which affects the composites' reinforcement. Indeed, for CNT and nanoG, the mechanical reinforcement depends on the load direction: IP moduli $\left(G_{12}\right.$ and $\left.G_{21}\right)$ are remarkably different from TT moduli $\left(G_{31}\right.$ and $\left.G_{32}\right)$. On the contrary, CB-MS and CB-HS do not reveal large differences. Whatever the filler for each composite the two moduli measured in IP configuration $\left(G_{12}\right.$ and $\left.G_{21}\right)$ are superimposed, as theoretically expected from symmetry conditions. Moreover, also the two moduli measured in TT configuration $\left(G_{31}\right.$ and $\left.G_{32}\right)$ are very close to each other. For this reason in the following no distinction will be done between $G_{12}$ and $G_{21}$ or between $G_{31}$ and $G_{32}$, but moduli will be generically indicated as GIP or GTT, respectively, unless otherwise specified.

These findings are indicative of transversally isotropic solids, i.e. materials whose properties measured in a plane are equal along any direction and are different from those measured perpendicularly to the plane. This result could be interpreted as a consequence of the organization of the filler particles inside the rubber matrix. Anisometric particles have a preferential orientation: they lie inside layers parallel to each other.
This picture of the composites, shown in Figure 2, was revealed by TEM images taken to CNT and nanoG filled systems, as discussed below in the text. A quantitative index of the composites' mechanical response, aimed at indicating the mechanical anisotropy, was called anisotropy index and was defined as the ratio of the property values in IP over the values in TT configuration. For each configuration, at least four repetitions were performed, and mean value and standard deviation are reported in Table 2. The standard deviation of the anisotropy index is calculated according to the rules of error propagation. In Figure 3 and Table 2 the effects of the different fillers on the behavior of the composites are clearly noticeable. Particles with a remarkable shape aniso effects, while more isometric particles such as CBMS and CB-HS lead to isotropic composites.

A remarkable level of anisotropy was calculated for both nanoG and CNT filled systems, although the moduli values are much lower in the case of nanoG compared to CNT. In fact, nanoG and CNT filled composites show an anisotropy index of the $G_{0.025}^{\prime}$ modulus equal to 1.83 and 1.95 , respectively, in spite of the fact that modulus values for nanoG are $4.40 \mathrm{MPa}$ (IP) and 2.4 MPa (TT), much lower than those for CNT, equal to $20.5 \mathrm{MPa}$ (IP) and $10.5 \mathrm{MPa}$ (TT).

The anisotropy index of the two types of $\mathrm{CB}$ is slightly larger than 1, 1.10 and 1.06 for CB-MS and for CBHS, respectively. Such (slight) deviation from isotropy could be due to an inhomogeneous filler distribution and dispersion, or to a slight shape anisotropy of filler aggregates. In a previous work it was observed [44] that CB generally exhibits a reduction of aggregate breadth, or 'flatness', in one direction. Some works [45-53] evidenced slightly anisotropic properties in elastomeric systems, in both compression and injection molded rubber parts. The orientation of molecules during the mould-filling operation was identified as the dominating factor, and the presence of CB-MS was found to increase the anisotropy [54]. 
Analogous findings were obtained for energy dissipation. The two fillers with anisometric particles, CNT and nanoG, gave the highest anisotropy index for $G^{\prime \prime}{ }_{\text {max }}$, irrespectively of the absolute value of $G^{\prime \prime}{ }_{\max }$, which is much higher in the case of CNT. Interestingly, the values of anisotropy index of $G_{0.025 \%}^{\prime}$ and of $G^{\prime \prime}{ }_{\text {max }}$ are very similar in the same nanocomposite. It seems therefore that reinforcement at small strain and energy dissipation are similarly affected by the filler loading direction.

Curves reported in Figure 3a allow a direct comparison between composites, CNT-35 and CB-HS-35, which achieve the largest moduli values. It is worth noting that, in TT configuration, the modulus of CBHS-35 at low strain amplitude is much larger than the modulus of CNT-35. This finding should be expected, taking into consideration the larger surface area of CB-HS. It is also worth observing that the moduli of CB-HS-35 in IP and TT configurations have almost the same values. The behavior of CBHS is indeed similar to the behavior of CB-MS: fillers with nearly isometric particles do not give rise to anisotropic mechanical properties. In the case of CNT35 , the enhancement of moduli values, from TT to IP configuration, could be attributed to the anisometry of the particles which build up CNT. Finally, it can be noted that the modulus reduction with shear strain amplitude for CNT-35 is slightly larger than that of CB-HS-35 (CB-HS-35 curves were interrupted at about 3\% strain amplitude). All these findings indicate the different mechanisms for the reinforcement promoted by the two fillers.

\subsubsection{Effect of filler content on the anisotropic mechanical behaviour}

The effect of filler content on the anisotropic behavior of NR based composites was investigated, for composites based on anisometric fillers such as CNT and nanoG. Composites were prepared with 35,15 and $4 \mathrm{phr}$ of nanofiller and their behavior was compared with the one of the neat NR matrix. In the composites, the fillers were considered to be either below or above their percolation threshold, i.e. the minimum filler amount required to establish a continuous filler network. Percolation thresholds of CNT and nanoG were reported [11] for composites based on synthetic poly(1,4-cis-isoprene): they were between 7 and $9 \mathrm{phr}$ for CNT and between 17 and $21 \mathrm{phr}$ for nanoG. The aim was to verify if the anisotropic effect could be dependent on filler percolation. In pre- vious papers by some of the authors, the mechanical percolation threshold in polyisoprene rubber was estimated, following an approach based on the Huber and Vilgis plot [56], in a range between 7 and $9 \mathrm{phr}$ for CNT [13, 55] and between 17 and $21 \mathrm{phr}$ for nanoG $[11,13]$.

The dependence of storage modulus $G^{\prime}$ on the strain amplitude for the neat NR matrix, nanoG-4 and nanoG-15 are reported in Figure 4, respectively, whereas the curves of nanoG-35 were already reported in Figure $3 b$. Table 3 shows the storage modulus
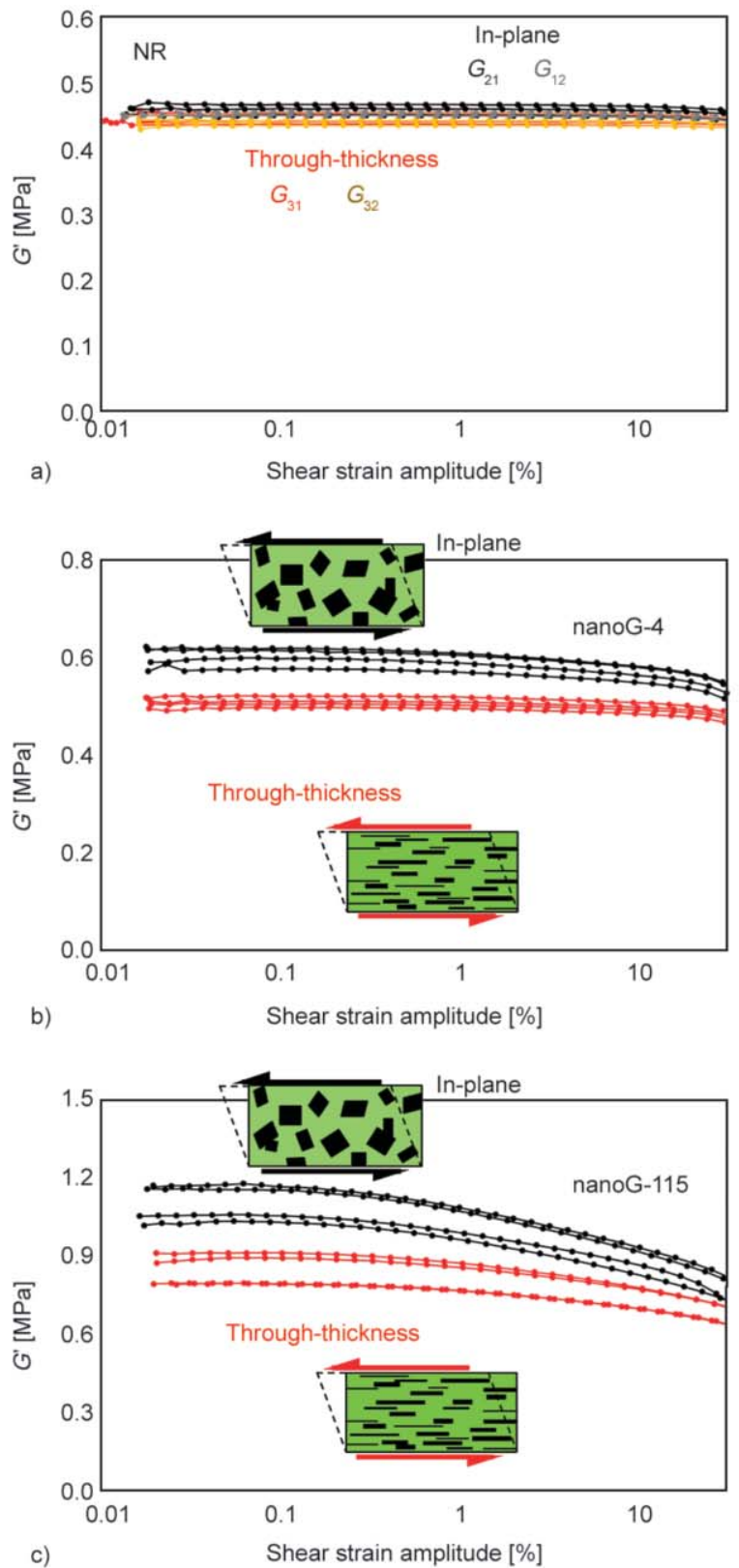

Figure 4. Storage moduli, measured in IP and TT configurations, vs shear strain amplitude, obtained from tests carried out on (a) the neat NR matrix and on composites filled with (b) $4 \mathrm{phr}$ and (c) $15 \mathrm{phr}$ of nanoG. 
Table 3. Average values of storage modulus $G^{\prime}$ at minimum $(0.025 \%)$ shear strain amplitude, and of maximum value of loss modulus $\left(G^{\prime \prime}{ }_{\max }\right)$, both in IP and TT configuration, and of anisotropy index for the composites with 4 and $15 \mathrm{phr}$ of nanoG, CNT or CB-MS.

\begin{tabular}{|l|c|c|c|c|c|c|}
\hline & nanoG-4 & nanoG-15 & CNT-4 $^{*}$ & CNT-15 $^{*}$ & CB-MS-4 $^{*}$ & CB-MS-15 $^{*}$ \\
\hline$G_{\text {IP, } 0.025 \%}^{\prime}[\mathrm{MPa}]$ & $0.60 \pm 0.02$ & $1.10 \pm 0.07$ & $1.12 \pm 0.02$ & $3.6 \pm 0.2$ & $0.505 \pm 0.002$ & $0.72 \pm 0.02$ \\
\hline$G_{\text {TT, } 0.025 \%}[\mathrm{MPa}]$ & $0.51 \pm 0.01$ & $0.85 \pm 0.06$ & $0.59 \pm 0.04$ & $1.61 \pm 0.09$ & $0.469 \pm 0.003$ & $0.644 \pm 0.005$ \\
\hline Anisotropy index $\left(G^{\prime}{ }_{0.025 \%}\right)$ & $1.19 \pm 0.03$ & $1.30 \pm 0.07$ & $1.88 \pm 0.04$ & $2.25 \pm 0.04$ & $1.077 \pm 0.006$ & $1.117 \pm 0.024$ \\
\hline$G_{\text {max }, \text { IP }[\mathrm{MPa}]}^{\prime \prime}$ & $0.042 \pm 0.005$ & $0.090 \pm 0.005$ & $0.092 \pm 0.005$ & $0.75 \pm 0.06$ & $0.0302 \pm 0.0014$ & $0.058 \pm 0.009$ \\
\hline$G_{\text {max }, \text { TT [MPa] }}^{\prime}$ & $0.032 \pm 0.005$ & $0.07 \pm 0.01$ & $0.043 \pm 0.002$ & $0.32 \pm 0.02$ & $0.0262 \pm 0.0006$ & $0.045 \pm 0.003$ \\
\hline Anisotropy index $\left(G^{\prime \prime}{ }_{\text {max }}\right)$ & $1.32 \pm 0.14$ & $1.33 \pm 0.09$ & $2.13 \pm 0.04$ & $2.35 \pm 0.04$ & $1.15 \pm 0.05$ & $1.27 \pm 0.13$ \\
\hline
\end{tabular}

*data from ref. [22]

values at low strain amplitude and the maximum value of loss modulus for both nanofillers and also for CB-MS N326 at 4 and $15 \mathrm{phr}$.

The level of reinforcement and the anisotropy levels of dynamic moduli in nanoG filled composites are clearly affected by the filler amount. As shown in Figure 3b, Figure 4, Table 2 and Table 3, by increasing nanoG content there is an increase of storage modulus and also of $G^{\prime \prime}$ max. The Payne effect is evident above the percolation threshold ( $35 \mathrm{phr}$, Figure $3 b$ ) and becomes appreciable when nanoG content approaches such threshold (15 phr, Figure 4c). Analogous findings can be seen in Figure 3 for CNT and $\mathrm{CB}$ based composites: the modulus non linearity is evident for composites with the carbon filler above its percolation threshold.

Both the storage moduli values at small strain and the corresponding anisotropy indexes are plotted in Figure 5 as a function of the filler content. As already commented, $G_{0.025}^{\prime}$ values of nanoG based composites are lower than those of the CNT based ones. Moreover, also the absolute values and dependence on the filler content of the anisotropy indexes are different. For CNT based composites, the anisotropy is remarkable already at low CNT content (4 phr),

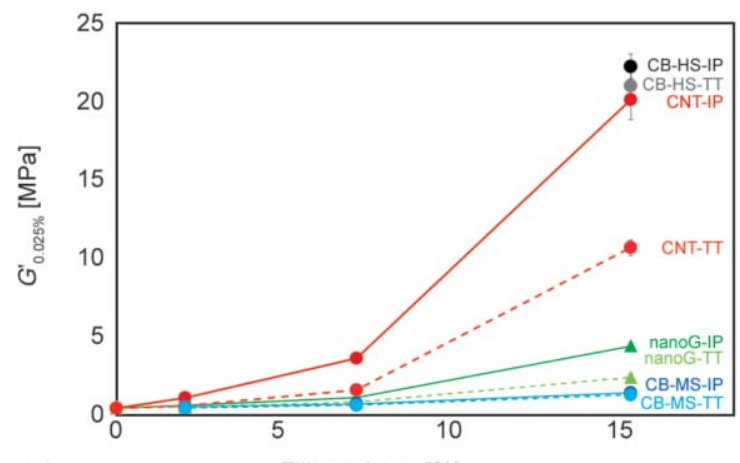

a)

Filler volume [\%] increases up to $15 \mathrm{phr}$ and decreases for larger CNT content. Such a dependence of the anisotropy index on the CNT content could be attributed to the high aspect ratio of CNT, which leads to high values at low CNT content, and to the tubes' bundles which, at high CNT content, hinder their orientation during the processing. Indeed, the anisotropy index of the CNT based composite decreases at contents higher than the percolation threshold.

On the other hand, the anisotropy index of nanoG increases almost linearly with the filler content. As commented above, nanoG achieves its percolation threshold at higher content, with respect to CNT. Moreover, nanoG cannot give rise to entangled structures. It could be also commented that a graphitic filler is known to act as a lubricant in a polymer composite. Hence, easier orientation, during processing, can be reasonably expected. Reduction of anisotropy index could be hypothesized also for nanoG based composites, at larger nanoG content. But such hypothesis cannot be verified or commented on the basis of the available experimental data.

CB-MS and CB-HS filled composites reveal a substantially isotropic behavior, whatever is the filler content and the level of reinforcement.

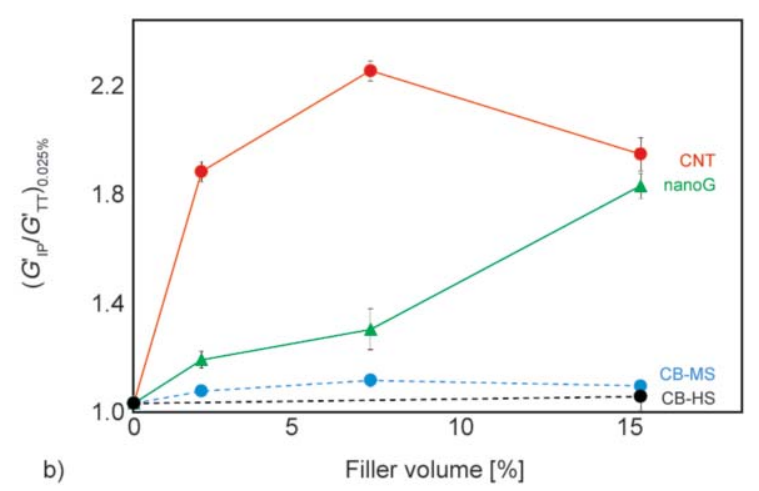

Figure 5. a) $G_{0.025 \%}^{\prime}$ moduli measured in IP and TT configuration and b) anisotropy index of $G_{0.025 \%}^{\prime}$ moduli as a function of filler content for fillers with different aspect ratio and geometry. (Ranking of filler aspect ratio: $\mathrm{CNT}>$ nanoG $>$ nearly isometric CB-MS $=\mathrm{CB}-\mathrm{HS}$ ) 
As already observed at $35 \mathrm{phr}$ of filler, also for the other filler contents the anisotropy of the energy dissipation, indicated by $G^{\prime \prime}{ }_{\max }$, is comparable to the anisotropy level of the initial storage modulus.

\subsection{TEM analysis of nanoG filled composites} TEM analyses were carried out on composites based on anisometric carbon fillers. Figure 6 and 7 show TEM micrographs of nanoG- 15 and nanoG-35, respectively. The observed samples are cut perpendicularly to the large surface of the rubber plate, in order to observe sections oriented parallel to axis 3 in Figure 2. In each figure, images taken at two different magnifications are shown together with the Selected Area Electron Diffraction pattern.

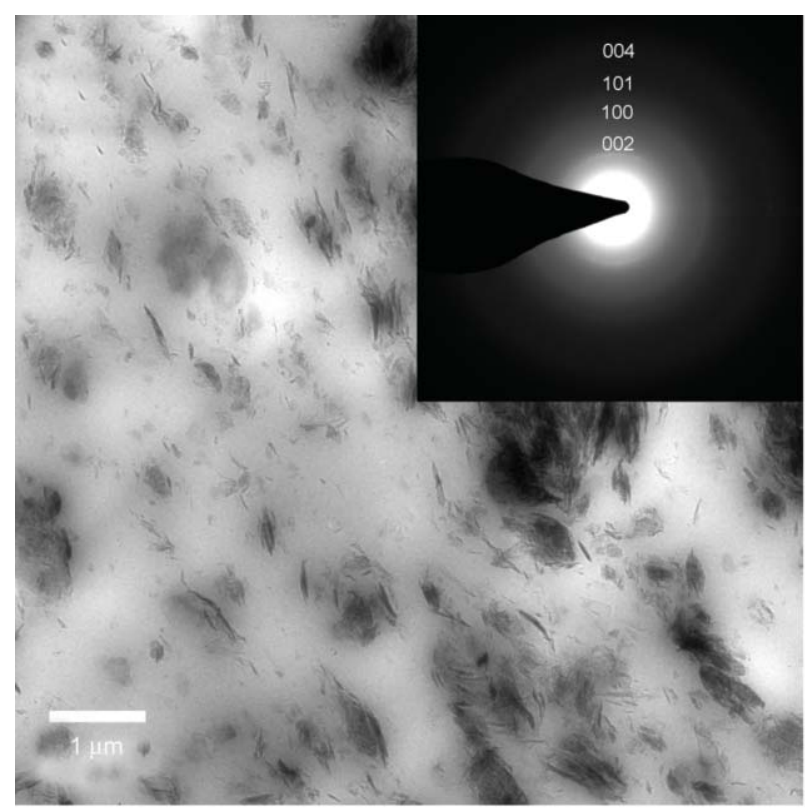

a)
In both Figure 6 and Figure 7, nano-sized stacks of graphite with dimension between 2 and $20 \mathrm{~nm}$ can be easily observed. In both cases, nanoG stacks appear grouped in aggregates with sizes between tens and hundreds of nanometers, uniformly distributed in the observed areas. However, whereas in nanoG15 the aggregates are separated from each other, in nanoG-35 the filler particles seem to form a nearly continuous network. In the images of both composites, most of the lamellae show their lateral thin side, and only a few lie flat in the sample slice. Moreover, a preferential orientation of the lamellae can be identified. In order to have an unbiased evaluation of such direction, SAED analyses were performed and correlated with the BF-TEM micrographs, following

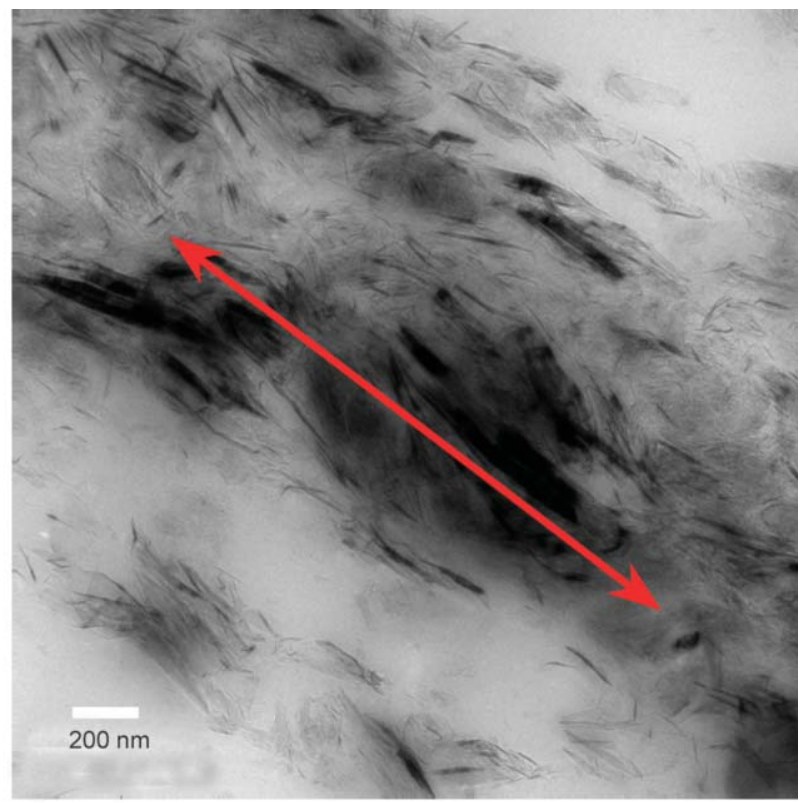

b)

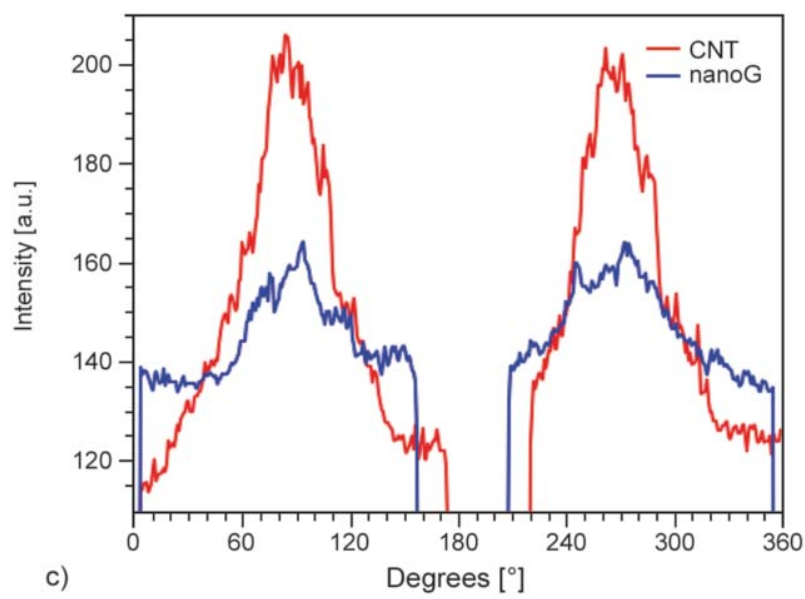

Figure 6. Bright field TEM micrographs of ultrathin slice of nanoG-15 nanocomposite at (a) low and (b) high magnification. The red arrow in (b) indicates the preferential orientation of nanoG particles identified by SAED analysis, whose indexed pattern is displayed in the inset. (c) Electron diffraction intensity profile as a function of azimuthal angle for the (002) Debye-Scherrer ring of nanoG-15 and CNT-15. 


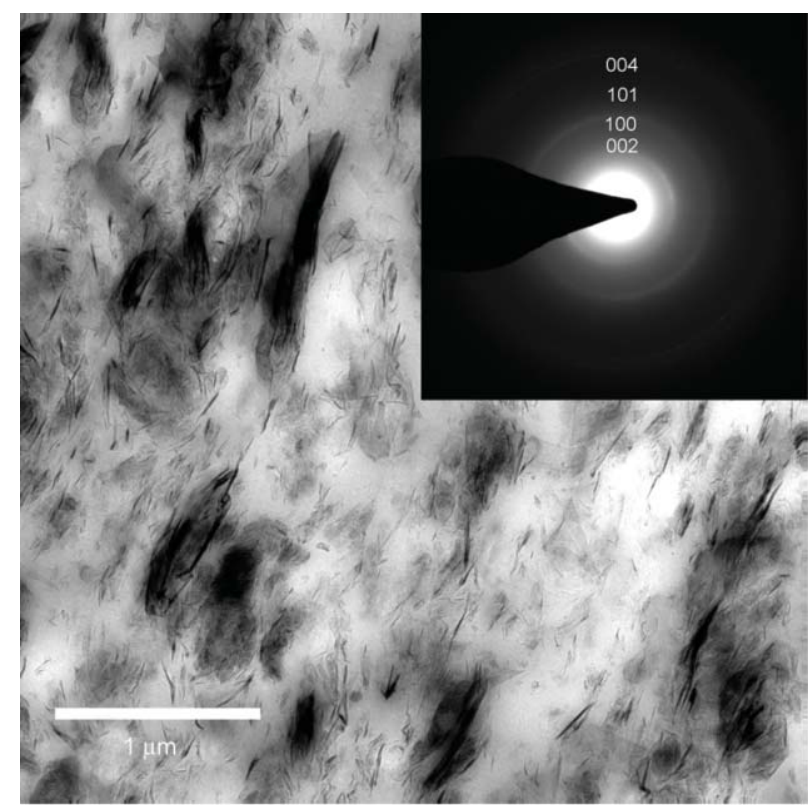

a)

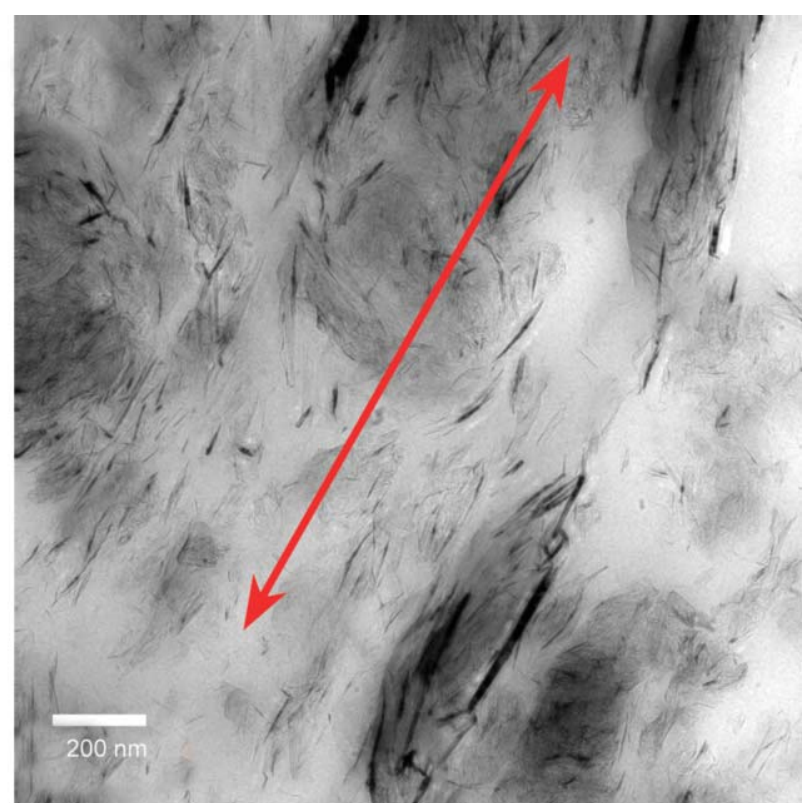

b)

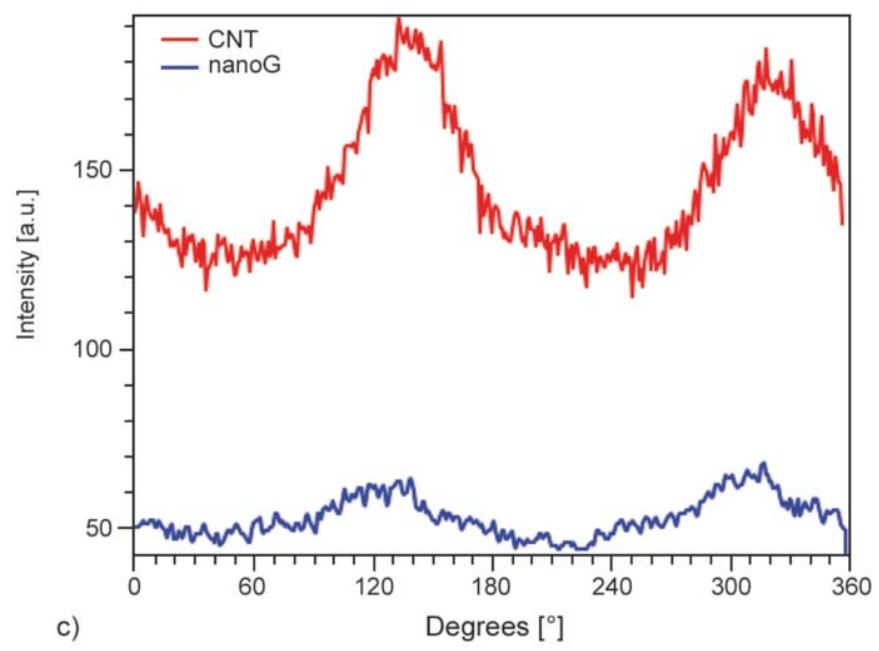

Figure 7. Bright field TEM micrographs of ultrathin slice of nanoG-35 nanocomposite at (a) low and (b) high magnification. The red arrow in (b) indicates the preferential orientation of nanoG particles identified by SAED analysis, whose indexed pattern is displayed in the inset. (c) Electron diffraction intensity profile as a function of azimuthal angle for the (002) Debye-Scherrer ring of nanoG-35 and CNT-35.

the same procedure already adopted for CNT based composites [22]. Quantitative evaluation of the degree of filler orientation is not possible, as it cannot be taken for granted that images, necessarily taken on a small area, are representative of the whole sample. However, preferential direction of orientation was identified by SAED: it is indicated, in the images, by a red arrow, which is perpendicular to axis 3 (see Figure 2). TEM investigation therefore confirms the hypothesis of filler stratification through the thickness of the rubber plate, as schematically represented in Figure 2.

Discussion on findings from TEM analysis of CNT based composites has been reported elsewhere [22].
For CNT-15 and CNT-35, TEM micrographs revealed a layered structuring of the nanofiller: bundles of CNT were mostly randomly distributed inside layers, disposed almost parallel to each other and perpendicular to the molding pressure. A few CNT connect the different layers through areas with lower amount of CNT. Also in the case of CNT filled systems, similarly to nanoG, SAED analysis revealed a preferential orientation of the filler particles, as can be observed in Figure $6 \mathrm{c}$ and $7 \mathrm{c}$, where the diffraction patterns of the composites based on either CNT or nanoG are compared. It is worth commenting that the intensity of the diffraction patterns does not provide useful quantitative information on the degree of 
orientation, mainly because the analysis was performed on a small area.

Composites' structure sketched in Figure 2 is thus confirmed by the results of TEM analysis and is in agreement with findings from dynamic-mechanical measurements: high shape anisotropy nanofillers impart a transversally isotropic mechanical behaviour to the elastomer based composites.

\subsection{Electrical resistivity measurements}

Elastomers are characterized by high electrical resistivity $\left(>10^{11} \Omega \cdot \mathrm{m}\right)$. Electrical conductivity $\sigma=1 / \rho$ can be increased with the incorporation of conductive fillers, such as carbon black or, generally, carbon based fillers. The electrical properties of the composites are affected by filler concentration, filler morphology, and filler dispersion. By increasing the filler content, the rubber composite exhibits a sharp variation of electrical properties, switching from an insulating to a conductive behaviour, at a filler amount termed percolation threshold. At this level, a continuous path of conductive filler particles is formed through the elastomer matrix, allowing the transport of charge carries which results in an electrical current $[57,58]$. Electrical resistivity was measured only at filler amounts above the percolation threshold.

In the present work, analogously to dynamic-mechanical tests, bulk electrical resistivity $\rho$ was measured on cubic specimens, cut from cured plates, both in IP and in TT configuration, with reference to the direction of current flow (see Figure 1c). In the TT configuration, the contacts were applied on faces $a$ and $a^{\prime}$, and the bias voltage was applied across the sheet thickness. TT configuration allowed the measurement of the resistivity offered by the specimen thickness, $\rho_{\mathrm{TT}}$. In the IP configuration, the contacts were applied on faces $b$ and $b^{\prime}$, and the bias voltage was applied across the 1-2 plane, along axis 1 or 2 . IP configuration allowed the measurement of the resistivity along two orthogonal directions in the sheet plane, $\rho_{\mathrm{IP} 1}$ and $\rho_{\mathrm{IP} 2}$. With reference to the specimens sketched in Figure 2, contacts were applied on the same surfaces where are the arrows representing the strains. Figure 2 shows the possible structuring of the fillers in the sections parallel to the current flow, in both test configurations. Such structuring occurs also in inner sections of the specimens, but for the sake of simplicity, only the outer layer was sketched.

Measurements were carried out on CB-MS, nanoG and CNT filled rubbers and also on the neat NR. The resistivity of CNT-4, CNT-15 and CNT-35, nanoG35, CB-MS-35 and CB-MS 50, measured both in IP and TT configurations, is shown in Figure 8: Figure $8 \mathrm{a}$ for CNT based composites, Figure $8 \mathrm{~b}$ for the other composites. The measurements revealed a linear $\mathrm{I}-\mathrm{V}$ dependence in the whole range of applied voltages. The electrical resistivity, $\rho$, was consequently calculated as: $\rho=R \cdot S / h$, where $R$ is the inverse of the slope of the best fitting linear approximation of the I-V characteristic, $S$ is the specimen cross-section perpendicular to the current and $h$ is the specimen height between the two contacts. Moreover, for some of the conductive samples, the resistivity was measured in IP configuration by applying a bias voltage across two different directions, 1 or 2 (as shown in Figure 1c) in order to assess possible anisotropic effects within the plane of the rubber plates. Since it was not measured any significant difference between the results obtained in IP configuration along different directions, these results are hereinafter reported as generically measured in 'IP configuration'.

It is worth noting that in the case of nanoG-4, nanoG15 , CB-MS-4, CB-MS-15 and NR the measured current was lower than $10^{-13} \mathrm{~A}$, indicating that these specimens are insulators. Hence, the resistivity was not calculated.

Only in the case of CNT filled systems it was possible to characterize the whole experimental range of filler contents. Figure 8a shows that the electrical resistivity decreases steadily by increasing the CNT content, and that $\rho_{\mathrm{TT}}$ is higher than $\rho_{\mathrm{IP}}$, irrespective of the filler amount. The conductivity in CNT filled rubbers is governed by tunneling of electrons between adjacent carbon nanotubes and through the thin polymer layer which separates them [58]. The lower resistivity at higher amounts of CNT could thus be interpreted as the result of the larger number of contact points between nanotubes and of the thinner elastomer layers separating adjacent nanotubes. Moreover, the fact that electrical conductivity is higher in IP than in TT configuration could be explained with the structuring of CNT filler [22], sketched also on the specimens drawn in Figure 8 . The conductivity is favored in IP configuration because the current flows through a well-connected random network of filler particles, whereas in TT configuration the current flows through alternate layers with different filler density. In other words, layers with lower amount of CNT connecting layers with higher amount of CNT increase the resistivity of the material. 

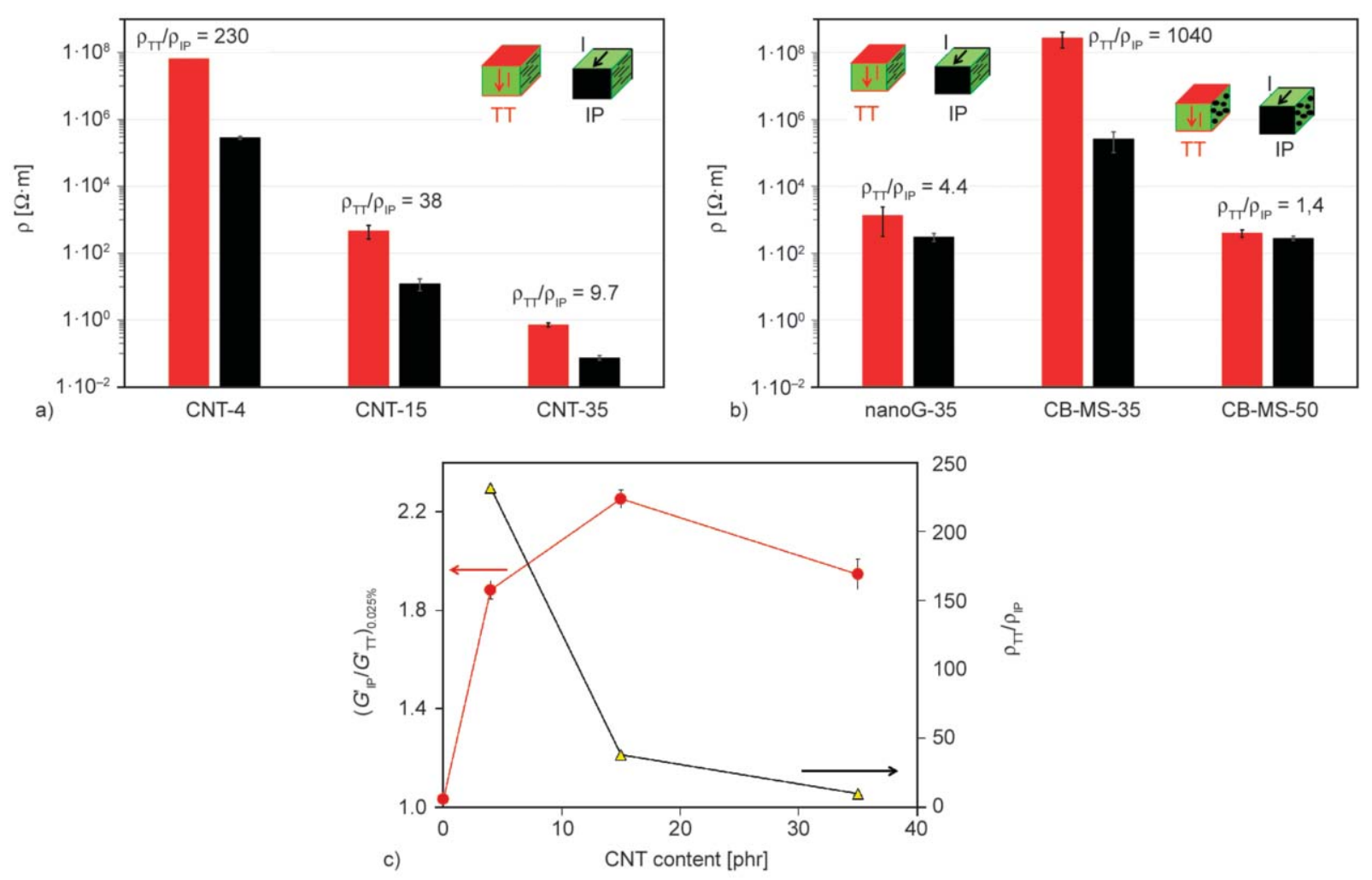

Figure 8. a) CNT-4, CNT-15 and CNT-35 and b) for nanoG-35, CB-MS-35, CB-MS-50. Anisotropy level ( $\left.\rho_{\mathrm{TT}} / \rho_{\mathrm{IP}}\right)$ of each sample is displayed above the bars. c) Comparison between meachanical $\left(\mathrm{G}_{\mathrm{II}} / \mathrm{G}_{\mathrm{TT}}^{\prime}\right.$ at $0.025 \%$ strain amplitude) and electrical $\left(\rho_{\mathrm{TT}} / \rho_{\mathrm{IP}}\right)$ anisotropy level of $\mathrm{C}_{\mathrm{NT}}$ filled composites..

The anisotropy level is calculated from the electrical properties as $\rho_{\mathrm{TT}} / \rho_{\mathrm{IP}}$, and the values are displayed in Figure $8 \mathrm{c}$. CNT filled composites show that the anisotropy level decreases by increasing the filler content: from 230 (at $4 \mathrm{phr}$ CNT) to 9.7 (at $35 \mathrm{phr}$ CNT). The high anisotropic level of CNT-4 could be due to the amount of CNT, which is very close to the one required to achieve percolation, i.e. to obtain a continuous filler network. As reported above, conductive properties can be imparted to the insulating NR matrix by achieving the percolation of the conductive filler. The electrical percolation should not be far from the mechanical percolation threshold, found to be in poly(isoprene) rubber in a range from 7 and $9 \mathrm{phr}[13,55]$. Therefore it could be argued that at a content of $4 \mathrm{phr}, \mathrm{CNT}$ are distributed in the whole (bulk) volume of the specimen but the connectivity between the CNT along the 'thickness' (TT, Figure $8 \mathrm{c}$ ) direction is different with respect to that along the 'in plane' (IP, Figure 8c) direction. The different connectivity results in a resistivity of the TT configuration much higher (two orders of magnitude) than that of the IP configuration. At $15 \mathrm{phr}$ as CNT content, the filler network should be fully established in any direction. The lower resistivity in IP configuration arises from the preferential orientation of the filler. The increase of CNT content from 15 to $35 \mathrm{phr}$ leads to a fourfold reduction of the anisotropy index. It can be hypothesized that at the maximum CNT content, the amount of conductive filler is such as to establish a continuous filler network in any direction, independently of any possible preferential filler orientation.

NanoG filled composites exhibited high electrical resistivity. The mechanical percolation threshold of nanoG in a synthetic poly(isoprene) rubber was measured in a range of $17-21 \mathrm{phr}[11,13]$. Only nanoG35 showed measurable values of resistivity (I $>$ $10^{-13} \mathrm{~A}$ ) because this is the only nanoG content above the electrical percolation threshold. This is confirmed also by the structural investigation by TEM analysis (see Figure 6 and 7): a nearly continuous path of filler particles can be observed at $35 \mathrm{phr}$, but not at $15 \mathrm{phr}$ of nanoG. Also for this nanofiller, TT resistivity is higher that IP resistivity, and also in this case the result is in agreement with the hypothesis of a layered structuring of the lamellar particles, observed in TEM images (Figure 6 and 7) and sketched in Figure 2. In the case of CB-MS filled composites, the resistivity could be measured only at $35 \mathrm{phr}$ of CB-MS, i.e. 
at a content slightly above the filler percolation threshold (mechanical percolation threshold of CB N326 in synthetic polyisoprene was reported in [13] at $29 \mathrm{phr}$ ). The electrical resistivity of CB-MS was much higher than that measured with the other nanofillers at the same filler amount, probably due to the lower filler specific surface area, which results in a much lower amount of filler-filler contact points. CB-MS was supposed to show low anisotropy effects because it is composed by nearly isometric particles. Strikingly, the electrical anisotropy index of CB-MS-35 is the highest measured in this work: $\rho_{\text {TT }}$ exceeds $\rho_{\text {IP }}$ by three orders of magnitude. Such high electrical anisotropy is close to the one observed for CNT-4. These experimental findings could be explained as follows. $35 \mathrm{phr}$ is an amount close to the CB-MS percolation threshold: the filler can form a continuous path through the composite, with flattened and denser filler areas lying in rubber plates. Such areas are not well connected among each other to create a continuous path also across the plate thickness. In order to further corroborate this result, a composite with $50 \mathrm{phr}$ as filler content was prepared and electrical resistivity was measured. The results are shown in Figure 8 b. The resistivity values of CB-MS-50 are lower than those of CB-MS-35, as expected, and are substantially independent of the measurement direction (anisotropy level $=1.4$ ). This result indicates that, when the amount of a nearly isometric filler is enough to establish a conductive continuous network throughout any direction, the filler does not provide anisotropic effects.

Electrical and mechanical anisotropy levels are differently influenced by CNT amount, as can be observed from the direct comparison shown in Figure $8 \mathrm{c}$. On one side mechanical anisotropy passes through a maximum: large CNT content, much above the percolation threshold, causes a reduction of filler orientation in CNT-35. On the other side, electrical anisotropy is maximum at $4 \mathrm{phr}$ CNT, then decreases steadily, due to the achievement of a homogenous conductive pathway. It is interesting to point out that the filler composition providing the maximum value of electrical anisotropy, CNT-4, does not correspond to the maximum value of mechanical anisotropy. This result was found also for CB-MS-35: the extremely high electrical anisotropy level (the highest among all the systems investigated) does not correspond to the nearly isotropic mechanical behavior. For both composites (CNT-4 and CB-MS-35) the filler amount is close to the percolation threshold of the filler. As already hypothesized, at such critical filler amount a continuous filler network seems to be established only on layers in the plane of the rubber plate and not perpendicularly to such planes. Since the establishment of a continuous conductive filler path remarkably affects the electrical conductivity of filled rubbers, high electrical anisotropy levels were obtained. The same result is not observed for mechanical anisotropy: the occurrence of a continuous filler network simply increases the rate of increase of mechanical stiffness with the filler amount, but the mechanical properties do not exhibit a discontinuity with the filler content at the percolation threshold, as in the case of electrical conductivity. Close to the filler percolation threshold, therefore, the filler structure has an amplifying effect on the electrical anisotropy level, but not on the mechanical one.

\section{Conclusions}

This work provides a quantitative investigation of anisotropic mechanical and electrical properties of NR composites filled with $\mathrm{sp}^{2}$ carbon allotropes, such as nearly isometric carbon black, carbon nanotubes and a lamellar nanosized graphite with high surface area, endowed with different surface area and geometrical aspect ratio. A complete mechanical characterization along all main directions could be performed by a non-standard testing approach. It is shown that the mechanical anisotropy is highly influenced by the filler aspect ratio. Composites with carbon black showed an isotropic mechanical behavior, independently of their surface area. Composites with anisometric particles, such as nanoG and CNT, revealed a transversal isotropic behavior, i.e. properties measured in the sheet plane (IP configuration) different from the properties measured perpendicularly to the sheet plane (TT configuration) and independent of the strain direction. The results are consistent with a layered structuring of the filler nanoparticles through the sheet thickness and with their random orientation inside the sheet plane, as confirmed by TEM analyses. The level of mechanical anisotropy consistently increases with the content of graphite nanoplatelets, which arrange themselves in parallel layers, hardly creating a network in the orthogonal direction. In the case of CNT, mechanical anisotropy increases with 
CNT amount and passes through a maximum at about 15 phr: large CNT contents give rise to connections in both orthogonal directions.

Electrical conductivity was observed already at $4 \mathrm{phr}$ for CNT based composites, whereas $35 \mathrm{phr}$ were required in the case of nanoG and CB-MS. Composites reveal anisotropic electrical properties. Anisotropy index consistently decreases for CNT based composites: once established an homogenous conductive pathway, CNT preferential orientation plays a negligible effect. CB-MS based composites revealed dramatic electrical anisotropy at $35 \mathrm{CB}-\mathrm{MS} \mathrm{phr}$, a CB-MS content close to the mechanical percolation threshold, suggesting that CB-MS aggregates create a continuous pathway first in the sheet plane.

The results here presented pave the way for the design of advanced soft composites materials, with selective mechanical and electrical properties along specific directions.

\section{Acknowledgements}

The authors thank Dr. Giovanna Cornacchia and Dr. Michela Faccoli of the University of Brescia for the preparation of samples for electrical measurements, and Ario Fahimi for the support in performing dynamic-mechanical tests.

\section{References}

[1] Donnet J. B., Custodero E.: Reinforcement of elastomers by particulate fillers. in 'The science and technology of rubber' (eds: Mark J. E., Erman B., Eirich F. R.), Academic Press, San Diego, 367-400 (2005).

[2] Zhao X.: Multi-scale multi-mechanism design of tough hydrogels: Building dissipation into stretchy networks. Soft Matter, 10, 672-687 (2014).

https://doi.org/10.1039/C3SM52272E

[3] Gerspacher M., Wampler W.: Fillers: Carbon black. in 'Basic elastomer technology' (eds.: Baranwal K. C., Stephens H. L.) Rubber Division of the ACS, New York, 57-81 (2001).

[4] Wang M-J., Gray C. A., Reznek S. A., Mahmud K., Kutsovsky Y.: Carbon black. in 'Kirk-Othmer encyclopedia of chemical technology' (ed.: Seidel A.) Wiley, New York, 761-803 (2004).

https://doi.org/10.1002/0471238961.0301180204011414. a01.pub2

[5] Thomas S., Maria H. J.: Progress in rubber nanocomposites. Woodhead, Cambridge (2017).

[6] Maiti M., Bhattacharya M., Bhowmick A. K.: Elastomer nanocomposites. Rubber Chemistry and Technology, 81, 384-469 (2008).

https://doi.org/10.5254/1.3548215
[7] Galimberti M., Cipolletti V., Musto S., Cioppa S., Peli G., Mauro M., Guerra G., Agnelli S., Riccò T., Kumar $\mathrm{V}$.: Recent advancements in rubber nanocomposites. Rubber Chemistry and Technology, 87, 417-442 (2014). https://doi.org/10.5254/rct.14.86919

[8] Bokobza L.: Multiwall carbon nanotube elastomeric composites: A review. Polymer, 48, 4907-4920 (2007). https://doi.org/10.1016/j.polymer.2007.06.046

[9] Bhattacharya M., Maiti M., Bhowmick A. K.: Tailoring properties of styrene butadiene rubber nanocomposite by various nanofillers and their dispersion. Polymer Engineering and Science, 49, 81-98 (2009). https://doi.org/10.1002/pen.21224

[10] Al-solamy F. R., Al-Ghamdi A. A., Mahmoud W. E.: Piezoresistive behavior of graphite nanoplatelets based rubber nanocomposites. Polymers for Advanced Technologies, 23, 478-482 (2012).

https://doi.org/10.1002/pat.1902

[11] Galimberti M., Kumar V., Coombs M., Cipolletti V., Agnelli S., Pandini S., Conzatti L.: Filler networking of a nanographite with a high shape anisotropy and synergism with carbon black in poly(1,4-cis-isoprene)based nanocomposites. Rubber Chemistry and Technology, 87, 197-218 (2014).

https://doi.org/10.5254/rct.13.87903

[12] Galimberti M., Cipolletti V., Coombs M., Riccò T., Agnelli S., Pandini S.: The role of nanofillers in promoting hybrid filler networking and synergism with carbon black in a hydrocarbon rubber. Kautschuk Gummi Kunstoffe, 66, 31-36 (2013).

[13] Agnelli S., Cipolletti V., Musto S., Coombs M., Conzatti L., Pandini S., Riccò T., Galimberti M.: Interactive effects between carbon allotrope fillers on the mechanical reinforcement of polyisoprene based nanocomposites. Express Polymer Letters, 8, 436-449 (2014). https://doi.org/10.3144/expresspolymlett.2014.47

[14] Agnelli S., Baldi F., Riccò T.: A tentative application of the energy separation principle to the determination of the fracture resistance (JIc) of rubbers. Engineering Fracture Mechanics, 90, 76-88 (2012).

https://doi.org/10.1016/j.engfracmech.2012.04.020

[15] Galimberti M., Infortuna G., Guerra S., Barbera V., Agnelli S., Pandini S.: $\mathrm{sp}^{2}$ carbon allotropes in elastomer matrix: From master curves for the mechanical reinforcement to lightweight materials. Express Polymer Letters, 12, 265-283 (2018).

https://doi.org/10.3144/expresspolymlett.2018.24

[16] Galimberti M., Barbera V., Guerra S., Conzatti L., Castiglioni C., Brambilla L., Serafini A.: Biobased Janus molecule for the facile preparation of water solutions of few layer graphene sheets. RSC Advances, 5, 8114281152 (2015).

https://doi.org/10.1039/C5RA11387C 
[17] Barbera V., Porta A., Brambilla L., Guerra S., Serafini A., Valerio A. M., Vitale A., Galimberti M.: Polyhydroxylated few layer graphene for the preparation of flexible conductive carbon paper. RSC Advances, 6, 87767-87777 (2016).

https://doi.org/10.1039/C6RA19078B

[18] Machado G., Chagnon G., Favier D.: Theory and identification of a constitutive model of induced anisotropy by the mullins effect. Journal of the Mechanics and Physics of Solids, 63, 29-39 (2014).

https://doi.org/10.1016/j.jmps.2013.10.008

[19] Rebouah M., Machado G., Chagnon G. D., Favier D.: Anisotropic Mullins stress softening of a deformed silicone holey plate. Mechanics Research Communications, 49, 36-43 (2013).

https://doi.org/10.1016/j.mechrescom.2013.02.002

[20] Kahraman H., Haberstroh E.: Mechanical characterisation of anisotropic stress softening in carbon black filled rubber. Plastics, Rubber and Composites, 42, 19-25 (2013). https://doi.org/10.1179/1743289812Y.0000000026

[21] Machado G., Chagnon G., Favier D.: Induced anisotropy by the Mullins effect in filled silicone rubber. Mechanics of Materials, 50, 70-80 (2012).

https://doi.org/10.1016/j.mechmat.2012.03.006

[22] Agnelli S., Pandini S., Serafini A., Musto S., Galimberti M.: Anisotropic nonlinear mechanical behavior in carbon nanotubes/poly(1,4-cis-isoprene) nanocomposites. Macromolecules, 49, 8686-8696 (2016).

https://doi.org/10.1021/acs.macromol.6b01682

[23] Ogawa M., Kudo T.: High-performance pneumatic tire having bead fillers of anisotropic elasticity. US 4711285 A (1987).

[24] Ponnamma D., Sadasivuni K. K., Grohens Y., Guo Q., Thomas S.: Carbon nanotube based elastomer composites - An approach towards multifunctional materials. Journal of Materials Chemistry C, 2, 8446-8485 (2014). https://doi.org/10.1039/c4tc01037j

[25] Voge C. M., Kariolis M., MacDonald R. A., Stegemann J. P.: Directional conductivity in SWNT-collagen-fibrin composite biomaterials through strain-induced matrix alignment. Journal of Biomedical Materials Research Part A, 86, 269-277 (2008).

https://doi.org/10.1002/jbm.a.32029

[26] Chau M., De France K. J., Kopera B., Machado V. R., Rosenfeldt S., Reyes L., Chan K. J. W., Förster S., Cranston E. D., Hoare T., Kumacheva E.: Composite hydrogels with tunable anisotropic morphologies and mechanical properties. Chemistry of Materials, 28, 3406-3415 (2016). https://doi.org/10.1021/acs.chemmater.6b00792

[27] Gao Y., Wu Y., Liu J., Zhang L.: Controlling the electrical conductive network formation of polymer nanocomposites via polymer functionalization. Soft Matter, 12, 9738-9748 (2016).

https://doi.org/10.1039/c6sm02201d
[28] Kueseng P., Sae-oui P., Sirisinha C., Jacob K., Rattanasom N.: Anisotropic studies of multi-wall carbon nanotube (MWCNT)-filled natural rubber (NR) and nitrile rubber (NBR) blends. Polymer Testing, 32, 1229-1236 (2013).

https://doi.org/10.1016/j.polymertesting.2013.07.005

[29] Mauro M., Cipolletti V., Galimberti M., Longo P., Guerra G.: Chemically reduced graphite oxide with improved shape anisotropy. The Journal of Physical Chemistry C, 116, 24809-24813 (2012).

https://doi.org/10.1021/jp307112k

[30] Agarwal B. D., Broutman L. J., Chandrashekhara K.: Analysis and performance of fiber composites. Wiley, New York (2006).

[31] Payne A. R., Whittaker R. E.: Low strain dynamic properties of filled rubbers. Rubber Chemistry and Technology, 44, 440-478 (1971).

https://doi.org/10.5254/1.3547375

[32] Robertson C. G., Roland C. M.: Glass transition and interfacial segmental dynamics in polymer-particle composites. Rubber Chemistry and Technology, 81, 506522 (2008).

https://doi.org/10.5254/1.3548217

[33] Bohm G. A., Tomaszewski W., Cole W., Hogan T.: Furthering the understanding of the non linear response of filler reinforced elastomers. Polymer, 51, 2057-2068 (2010).

https://doi.org/10.1016/j.polymer.2010.01.047

[34] Heinrich G., Klüppel M.: Recent advances in the theory of filler networking in elastomers. in 'Filled elastomers drug delivery systems' (eds.: Abe A., Albertsson A-C., Cantow H-J., Dusek K., Höcker H., Joanny J-F., Kausch H-H., Lee K-S., Monnerie L.) Volume 160, Springer, Berlin, 1-44 (2002). https://doi.org/10.1007/3-540-45362-8 1

[35] Maier P. G., Goritz D.: Molecular interpretation of the Payne effect. Kautschuk Gummi Kunststoffe, 49, 1821 (1996).

[36] Chazeau L., Brown J. D., Yanyo L. C., Sternstein S. S.: Modulus recovery kinetics and other insights into the Payne effect for filled elastomers. Polymer Composites, 21, 202-222 (2000). https://doi.org/10.1002/pc. 10178

[37] Sternstein S. S., Zhu A-J.: Reinforcement mechanism of nanofilled polymer melts as elucidated by nonlinear viscoelastic behavior. Macromolecules, 35, 7262-7273 (2002).

https://doi.org/10.1021/ma020482u

[38] Montes H., Lequeux F., Berriot J.: Influence of the glass transition temperature gradient on the nonlinear viscoelastic behavior in reinforced elastomers. Macromolecules, 36, 8107-8118 (2003).

https://doi.org/10.1021/ma0344590

[39] Zhu Z., Thompson T., Wang S-Q., von Meerwall E. D., Halasa A.: Investigating linear and nonlinear viscoelastic behavior using model silica-particle-filled polybutadiene. Macromolecules, 38, 8816-8824 (2005).

https://doi.org/10.1021/ma050922s 
[40] Kalfus J., Jancar J.: Elastic response of nanocomposite poly(vinylacetate)-hydroxyapatite with varying particle shape. Polymer Composites, 28, 365-71 (2007). https://doi.org/10.1002/pc.20273

[41] Jancar J., Douglas J. F., Starr F. W., Kumar S. K., Cassagnau P., Lesser A. J., Stermstein S. S., Buehler M. J.: Current issues in research on structure-property relationships in polymer nanocomposites. Polymer, 51, 3321-3343 (2010).

https://doi.org/10.1016/j.polymer.2010.04.074

[42] Funt J. M.: Dynamic testing and reinforcement of rubber. Rubber Chemistry and Technology, 61, 842-865 (1988).

https://doi.org/10.5254/1.3536222

[43] Gauthier C., Reynaud E., Vassoille R., Ladouce-Stelandre L.: Analysis of the non-linear viscoelastic behaviour of silica filled styrene butadiene rubber. Polymer, 45, 2761-2771 (2004). https://doi.org/10.1016/j.polymer.2003.12.081

[44] Gruber T. C., Zerda T. W., Gerspacher M.: 3D morphological characterization of carbon-black aggregates using transmission electron microscopy. Rubber Chemistry and Technology, 67, 280-287 (1994). https://doi.org/10.5254/1.3538674

[45] Gurney W. A., Gough V. E.: Moulding anisotropy. Rubber Chemistry and Technology, 20, 863-878 (1947). https://doi.org/10.5254/1.3543312

[46] Blow C. M., Demirli H. B., Southwart D. W.: Anisotropy in molded nitrile rubber. Journal of the Institution of the Rubber Industry, 8, 244-248 (1974).

[47] Chang W. V., Yang P. H., Salovey R.: Mold-flow-induced anisotropy in nitrile rubber. Rubber Chemistry and Technology, 54, 449-459 (1981).

https://doi.org/10.5254/1.3535817

[48] Hamed G. R.: Anisotropy induced during compression molding of rubber. Journal of Applied Polymer Science, 27, 4081-4083 (1982). https://doi.org/10.1002/app.1982.070271036

[49] Nakashima K., Fukuta K., Mineki M.: Anisotropic shrinkage of injection-molded rubber. Journal of Applied Polymer Science, 17, 769-778 (1973).

https://doi.org/10.1002/app.1973.070170309
[50] Filippova T. I., Bartenev G. M.: Determination of the structural anisotropy of molded vulcanizates by swelling. International Polymer Science and Technology, 2, 2122 (1975).

[51] Tsai B. C.: Injection molding of rubber: Structure, processing and properties. Rubber Chemistry and Technology, 51, 26-34 (1978).

https://doi.org/10.5254/1.3535724

[52] Wheelans M. A.: Injection molding of rubber. Rubber Chemistry and Technology, 51, 1023-1043 (1978). https://doi.org/10.5254/1.3535769

[53] Deng J. S., Isayev A. I.: Injection molding of rubber compounds: Experimentation and simulation. Rubber Chemistry and Technology, 64, 296-324 (1991). https://doi.org/10.5254/1.3538560

[54] Lavebratt H., Stenberg B.: Anisotropy in injectionmolded styrene-butadiene rubbers. 1. Plastics Rubber and Composites Processing and Applications, 20, 3-13 (1993).

[55] Galimberti M., Coombs M., Riccio P., Riccò T., Passera S., Pandini S., Conzatti L., Ravasio A., Tritto I.: The role of CNTs in promoting hybrid filler networking and synergism with carbon black in the mechanical behavior of filled polyisoprene. Macromolecular Materials and Engineering, 298, 241-251 (2013). https://doi.org/10.1002/mame.201200075

[56] Huber G., Vilgis T. A.: Universal properties of filled rubbers: Mechanisms for reinforcement on different length scales. Kautschuk Gummi Kunstoffe, 52, 102107 (1999).

[57] Bokobza L.: Mechanical, electrical and spectroscopic investigations of carbon nanotube-reinforced elastomers. Vibrational Spectroscopy, 51, 52-59 (2009). https://doi.org/10.1016/j.vibspec.2008.10.001

[58] Lorenz H., Fritzsche J., Das A., Stöeckelhuber K. W., Jurk R., Heinrich G., Klüppel M.: Advanced elastomer nano-composites based on CNT-hybrid filler systems. Composites Science and Technology, 69, 2135-2143 (2009).

https://doi.org/10.1016/j.compscitech.2009.05.014 